\section{Emergency contraception prescribing in a GUM clinic: missed opportunities for improving sexual and reproductive health}

Since publication of the Sexual Health Strategy in England, ${ }^{1}$ integration of UK sexual health services has been increasingly promoted. Such services may increase potential for screening and prevention, improve continuity of care and facilitate more efficient resource use. ${ }^{2}$ One concern about integration has been the need for further staff training. ${ }^{23}$ However, integration may also be advantageous for training, facilitating skill sharing between professionals. The results of an audit of emergency contraception (EC) prescribing in the Edinburgh Genitourinary Medicine (GUM) clinic highlight the need for encouragement of such skill sharing.

In 2006 the Faculty of Family Planning and Reproductive Health Care (now the Faculty of Sexual and Reproductive Healthcare) Clinical Effectiveness Unit published UK guidance on the prescribing of EC. ${ }^{4}$ At the time of the audit EC was prescribed in our GUM clinic as levonorgestrel (LNG) with referral to the local family planning clinic (FPC) for intrauterine device (IUD) insertion. The audit determined whether our prescribing of LNG was in line with current guidance.

The case notes of all women prescribed LNG for EC in the Edinburgh GUM clinic over a 24-month period ending 30 June 2009 were reviewed by a GUM registrar. This established women's current method of contraception, reasons for requiring EC, whether IUD was discussed as alternative EC, whether a sexual history was taken and sexually transmitted infection (STI) screening offered, and whether options for future contraception were discussed.

A total of 293 women were prescribed LNG during the audit period; 234 (80\%) women were aged 25 years and under (mean age 23, range 14-49, years). The demographics of women attending for EC mirrored those attending the GUM service in general.

IUD insertion was discussed as an alternative EC with only 53 (18\%) women.

Seventy-one percent (208) of women presenting for EC used condoms for contraception, $14 \%$ (41) the oral contraceptive pill (OCP) and 15\% (44) no contraception (Table 1).

All the women had a sexual history taken and $24 \%$ (70) reported a previous STI. Some $71 \%$ (208) were offered STI screening within 2 weeks of LNG
Table 1 Women's reasons for requiring emergency contraception

\begin{tabular}{lc}
\hline Reason for EC & Women $[\boldsymbol{n}(\%)]$ \\
\hline No condom & $141(48)$ \\
Condom burst & $81(28)$ \\
Condom came off & $30(10)$ \\
Missed OCP & $33(11)$ \\
Antibiotics with OCP & $8(3)$ \\
\hline
\end{tabular}

EC, emergency contraception; $\mathrm{OCP}$, oral contraceptive pill.

prescribing; $57 \%$ (119) of these accepted. Twelve $(10 \%)$ of the women screened were diagnosed with a STI $(9 \%$ chlamydia, $1 \%$ warts). Eighty-three percent of those diagnosed with STIs were aged under 25 years (i.e. 10 women).

Eighty-eight $(30 \%)$ women attending for $\mathrm{EC}$ received no advice on future contraception; $52 \%$ (152) were advised to attend the FPC to discuss contraception, $15 \%$ (44) continued on the OCP and 3\% (9) of women were started on the OCP in clinic.

Most women attending clinic for EC were using inadequate or no contraception and onward contraceptive advice was poor. Since this audit relied on accurate documentation of EC consultations by clinicians it is possible that the results partially reflect poor documentation rather than solely poor advice giving. However, the results clearly illustrate room for improvement and highlight a need for training of GUM professionals in aspects of reproductive health.

In my opinion the need for provision of reliable onward contraception and benefits of STI screening in those presenting for EC support increased integration of sexual health services to promote skill sharing. Further studies of EC prescribing in non-integrated GUM clinics would be valuable and may demonstrate significant variation in onward contraception advice and provision. The Edinburgh family planning and GUM services have recently merged to form a fully integrated service. I look forward to completion of the audit cycle in our new service to determine whether integration has been associated with improved care for this group.

Lisa Goodall BMBCh, MRCP

Specialty Registrar in GUM, Chalmers Sexual Health Centre, Edinburgh, UK; lisagoodall@doctors.org.uk

\section{Competing interests None.}

J Fam Plann Reprod Health Care 2012;38:140. doi:10.1136/jprhc-2011-100241

\section{References}

1 Department of Health. Better Prevention, Better Services, Better Sexual Health. The National Strategy for Sexual Health and HIV. 2001. http://www.dh.gov.uk/en/

Publicationsandstatistics/Publications/
PublicationsPolicyAndGuidance/DH_4003133 [accessed 3 February 2012].

2 Kane R, Wellings K. Staff training in integrated sexual health services. Sex Transm Infect 2003;79:354-356.

3 Robinson G. Integrated sexual health: a better way of working? J Fam Plann Reprod Health Care 2009:35:249-250.

4 Faculty of Family Planning and Reproductive Health Care Clinical Effectiveness Unit. FFPRHC Guidance (April 2006). Emergency contraception. $J$ Fam Plann Reprod Health Care 2006:32:121-128. 